\title{
Le thème du travail agricole dans les tableaux des Glasgow Boys, ou l'Écosse selon des peintres victoriens
}

Farm Work in the Glasgow Boys' Paintings or Scotland As Seen by a Group of Victorian Painters

\section{Marion Amblard}

\section{OpenEdition \\ Journals}

Édition électronique

URL : http://journals.openedition.org/etudesecossaises/1114

DOI : 10.4000/etudesecossaises. 1114

ISSN : 1969-6337

\section{Éditeur}

UGA Éditions/Université Grenoble Alpes

\section{Édition imprimée}

Date de publication : 25 avril 2016

Pagination : 119-132

ISBN : $978-2-84310-324-7$

ISSN : $1240-1439$

\section{Référence électronique}

Marion Amblard, "Le thème du travail agricole dans les tableaux des Glasgow Boys, ou l'Écosse selon des peintres victoriens », Études écossaises [En ligne], 18 | 2016, mis en ligne le 01 janvier 2017, consulté le 16 mars 2021. URL : http://journals.openedition.org/etudesecossaises/1114 ; DOI : https://doi.org/10.4000/etudesecossaises.1114 


\section{Le thème du travail agricole dans les tableaux des Glasgow Boys, ou l'Écosse selon des peintres victoriens}

Tout au long de l'année 2010, les peintres de l'école de Glasgow, désormais connus sous le nom de Glasgow Boys, ont été à l'honneur en GrandeBretagne. Plusieurs expositions leur ont été consacrées à Londres, à Édimbourg et surtout dans la ville de Glasgow qui a organisé la plus grande rétrospective dédiée à ces artistes depuis $1968^{1}$. Les Glasgow Boys ont occupé le devant de la scène artistique écossaise des années 1880 jusqu'au début du $\mathrm{Xx}^{\mathrm{e}}$ siècle, ils jouissaient alors d'une renommée internationale puisque leurs tableaux étaient exposés en Europe et aux États-Unis. Après la Première Guerre mondiale, ce groupe de peintres est tombé dans l'oubli et ce ne fut qu'à partir de la fin des années 1960 que leur œuvre fut redécouverte et revalorisée. De nos jours, avec les Colourists ${ }^{2}$, ce sont indéniablement les peintres écossais les plus prisés en Grande-Bretagne ${ }^{3}$. On distingue plusieurs périodes dans leur œuvre : à leurs débuts, ils furent influencés par les artistes naturalistes ${ }^{4}$ et peignirent des tableaux

1. L'exposition qui s'intitule The Glasgow Boys a eu lieu à la Fleming Collection, à Londres, du 14 septembre 2010 au 18 décembre 2010. La National Gallery of Scotland a organisé l'exposition The Glasgow Boys: Drawing Inspiration du 29 mai au 15 septembre 2010. Pioneering Painters: The Glasgow Boys 1880-1900 a été tout d'abord présentée à Glasgow, à la Kelvingrove Art Gallery and Museum du 9 avril au 27 septembre 2010, puis a été exposée à la Royal Academy of Arts à Londres du 30 octobre 2010 au 23 janvier 2011.

2. Le groupe d'artistes désormais connu sous le nom de Colourists fut des plus actifs durant la période de l'entre-deux-guerres et se compose de quatre peintres : Francis Campbell Boileau Cadell (1883-1937), John Duncan Fergusson (1874-1961), George Leslie Hunter (1879-1931) et Samuel John Peploe (1871-1935). Sur les Colourists voir R. Billcliffe, 1989, The Scottish Colourists: Cadell, Fergusson, Hunter and Peploe, Londres, P. Long et E. Cumming, 2000, The Scottish Colourists, 1900-1930: F. C. B. Cadell, 7. D. Fergusson, G. L. Hunter, S. 7. Peploe, Édimbourg, National Galleries of Scotland.

3. Ces deux groupes d'artistes font l'objet du plus grand nombre d'expositions consacrées à des peintres écossais.

4. Le courant pictural naturaliste est défini comme étant «la représentation du quotidien avec un accent implacable de vérité. Le Naturalisme [...] est la description non flattée de la condition humaine, prenant pour cadre des lieux de travail ou de plaisir». («Le Naturalisme», Dictionnaire universel de l'art et des artistes, vol. 3, Paris, Fernand Hazan, 1967, p. 70) 
représentant les zones rurales de l'Écosse; à partir des années 1890, ils délaissèrent progressivement le naturalisme pour s'inspirer du style de James Abbott McNeill Whistler (1834-1903); par la suite, les Glasgow Boys se spécialisèrent dans des genres et des styles différents. Ainsi, Arthur Melville (1855-1904) réalisa un grand nombre d'aquarelles inspirées des paysages qu'il avait pu observer lors de ses séjours en Afrique du Nord. Également aquarelliste, Joseph Crawhall (1861-1913) se spécialisa dans la peinture des animaux tandis que Sir John Lavery (1856-1941) ${ }^{5}$ compta parmi les portraitistes les plus prisés de Grande-Bretagne. Dans cet article, nous allons plus précisément nous intéresser à la série de tableaux ayant pour thème le travail des ouvriers agricoles écossais qu'ils ont peints entre 1880 et le début des années 1890. Cette étude se propose de rappeler l'importance du thème du travail agricole au sein de l'œuvre des Glasgow Boys; elle démontrera aussi qu'avec ces toiles les peintres ont donné une vision de l'Écosse très différente de celle véhiculée dans les tableaux de la majeure partie des peintres écossais depuis les années 1820.

Il conviendra tout d'abord de présenter brièvement les Glasgow Boys, de revenir sur leurs relations avec leurs collègues de la Royal Scottish Academy et de replacer leur œuvre dans un contexte international, ceci afin de comprendre les raisons pour lesquelles le travail agricole fut un temps leur thème de prédilection. Les Glasgow Boys ont pour la plupart étudié sur le continent européen, le plus souvent en France. De retour en Écosse, ils s'inspirèrent du style et de l'approche des peintres français tels Jean-François Millet (1814-1875), Jules Breton (1827-1906) et Jules Bastien-Lepage (1848-1884), pour peindre des tableaux en réaction aux toiles des peintres de genre et des paysagistes membres de la Royal Scottish Academy. En mettant les scènes de genre paysannes des Glasgow Boys en relation avec les tableaux de leurs contemporains écossais, nous constaterons que ce ne fut pas seulement le style de leurs compatriotes qu'ils rejetaient, mais aussi leurs thèmes. Depuis les années 1820, les paysagistes avaient peint presque exclusivement des vues des Highlands. Avec leurs tableaux paysans, les peintres de l'école de Glasgow ont rappelé que l'Écosse se compose de deux régions, et non pas seulement des Highlands; ils ont aussi présenté l'Écosse comme une terre d'industrie, où le travail compte parmi les qualités fondatrices de l'identité écossaise.

5. Tous les peintres de l'école de Glasgow n'étaient pas natifs de cette ville, mais y ont ouvert un atelier; Joseph Crawhall et John Lavery venaient respectivement d'Angleterre et d'Irlande. Lavery habita à Glasgow entre 1885 et 1896, tandis que Crawhall y séjourna fréquemment jusqu'en 1898. À cette période, ils travaillèrent régulièrement avec les autres artistes appartenant au mouvement des Glasgow Boys. 
Le terme Glasgow Boys désigne une quinzaine de peintres qui eurent un atelier à Glasgow et partagèrent les mêmes modèles et les mêmes objectifs entre 1880 et $1900^{6}$. Ces artistes ne constituaient pas un groupe homogène puisqu'il est possible de distinguer en leur sein trois cercles de peintres : le premier comprenait William York MacGregor (1855-1923) et James Paterson (1854-1932) qui travaillèrent ensemble plusieurs étés sur la côte est de l'Écosse; le deuxième groupe se composait de James Guthrie (1859-1930), Joseph Crawhall, George Henry (1858-1943) et d'Edward Arthur Walton (1860-1922), artistes qui se lièrent d'une profonde amitié et se retrouvèrent régulièrement pour peindre en plein air; le troisième cercle de peintres se constituait d'Alexander Roche (18611921), John Lavery et de Thomas Millie Dow (1848-1919) qui firent connaissance lors de leurs études dans les ateliers parisiens et séjournèrent dans le village de Grez-sur-Loing. À ces artistes qui étaient les principaux représentants des Glasgow Boys, il convient d'ajouter Arthur Melville, Edward Atkinson Hornel (1864-1933), Alexander Mann (18531908), Robert Macaulay Stevenson (1854-1952), James Nairn (18591904) et David Gauld (1865-1936). L'ensemble de ces peintres avait le plus souvent l'occasion de se côtoyer l'hiver lorsqu'ils retournaient travailler dans leur atelier à Glasgow.

À leurs débuts, ces artistes avaient en commun une profonde admiration pour les peintres naturalistes français et partageaient la volonté de transformer la peinture écossaise. Tous s'étaient également heurtés à l'opposition des membres de la Royal Scottish Academy qui avaient refusé d'exposer leurs tableaux. Depuis sa création en 1826, cette institution avait tenu à l'écart les peintres travaillant à Glasgow; c'étaient presque exclusivement les artistes ayant un atelier à Édimbourg qui

6. David Martin fut le premier historien de l'art à reconnaître l'existence de l'école de peinture de Glasgow et à lui consacrer un ouvrage publié à la fin du XIX ${ }^{e}$ siècle. Selon Billcliffe il semblerait que ce soient les artistes constituant l'école de Glasgow qui soient à l'origine de l'appellation boys, car entre eux ils avaient pour habitude de se nommer ainsi : "Any artist from Glasgow was cited as a member of the Glasgow "School" by the local social magazines Quiz and The Bailie at the beginning of the 1880s. Recognition outside Glasgow of their work as having a certain unity came to the painters after an exhibition at the Grosvernor Gallery in London in 1890. It was at that time and by the London critics that the term "School" was coined; to the painters themselves and their friends in Glasgow they "were just the Boys". 》 (Billcliffe, 2002, p. 15) Les spécialistes sont en désaccord sur le nombre exact de peintres faisant partie des Glasgow Boys. Dans son ouvrage The Glasgow Boys. The Glasgow School of Painting 1875-1895, Roger Billcliffe compte quatorze peintres : Joseph Crawhall, Thomas Millie Dow, James Guthrie, George Henry, Edward Atkinson Hornel, William Kennedy (1859-1918), Sir John Lavery, William York MacGregor, Alexander Mann, Arthur Melville, James Paterson, Alexander Roche, Robert Macaulay Stevenson et Edward Arthur Walton. Quant à eux, les auteurs du catalogue de l'exposition Pioneering Painters. The Glasgow Boys 1880-1900 répertorient au total dix-huit peintres. Outre les peintres énoncés précédemment, ils considèrent que David Gauld, James Whitelaw Hamilton, James Nairn et James Stuart Park (1862-1933) étaient également membres des Glasgow Boys. 
avaient été élus membres de la Royal Scottish Academy et qui avaient pu y exposer leurs œuvres. De plus, dans un premier temps, les académiciens n'approuvèrent ni le style des peintres de Glasgow, ni les thèmes qu'ils retenaient pour leurs tableaux. Au début des années 1880, les membres de la Royal Scottish Academy spécialisés dans la peinture de genre suivaient la tradition de Sir David Wilkie (1785-1841), tandis que les paysagistes représentaient essentiellement des vues des Highlands. Depuis la fin des années 1820, la plupart des paysagistes imitait le style d'Horatio McCulloch (1805-1867) qui avait connu un immense succès auprès des collectionneurs avec ses tableaux de paysages des comtés du nord-ouest. Les peintres, qui jusque-là avaient exécuté le plus souvent des vues des Lowlands, délaissèrent les comtés du sud pour les Highlands afin de profiter de l'engouement collectif de leurs contemporains pour cette région ${ }^{7}$. Si les mécènes écossais avaient commandé peu de tableaux de paysages, à partir des années 1830, ils firent l'acquisition d'un grand nombre de peintures représentant les Highlands. Ces paysages peints reprenaient le plus souvent les mêmes éléments puisqu'ils représentaient traditionnellement de vastes étendues montagneuses et brumeuses peuplées de cerfs ou de moutons, avec parfois des personnages vêtus d'un kilt et les ruines d'un château visibles en arrière-plan. Ces nombreux tableaux ont diffusé une image romantique et idéalisée de la région qui servit à ancrer dans les esprits la nouvelle identité écossaise qui fut construite à l’issue des guerres napoléoniennes. Walter Scott, les hommes de lettres et les peintres élaborèrent une identité binationale à la fois écossaise et britannique dont l'une des particularités fut d'assimiler l'ensemble de l'Écosse aux Highlands afin d'insister sur la spécificité culturelle de l'Écosse et sur le fait que celle-ci était un partenaire égal à l'Angleterre au sein de la Grande-Bretagne ${ }^{8}$.

Lorsque les Glasgow Boys commencèrent à peindre, les membres de la Royal Scottish Academy acceptèrent difficilement d'exposer des tableaux non conformes aux traditions établies par Wilkie et McCulloch. Quelques années avant les peintres de Glasgow, Sir George Reid (1841-1913) avait déjà eu à faire face à l'hostilité des académiciens qui à l'occasion d'une exposition n'avaient pas hésité à apporter quelques retouches à l'une de ses toiles afin de la rendre, selon eux, plus acceptable ${ }^{9}$. Les académiciens

7. Les Highlands devinrent une destination des plus touristiques notamment en raison de l'engouement que suscitèrent la publication des poèmes d'Ossian de James Macpherson durant les années 1760, puis les œuvres de Walter Scott parues au début du XIX siècle.

8. Plusieurs ouvrages se sont intéressés à la définition de l'identité écossaise au début Xix ${ }^{\mathrm{e}}$ siècle. Entre autres, Devine et Pittock sont revenus en détail sur le contexte dans lequel l'identité prounioniste fut construite. Voir Devine (1999) et Pittock (2001).

9. Ainsi que l'explique le professeur John Morrison : «Reid had exhibited Spynie Palace and Loch at the RSA, Horatio McCulloch, the chief exponent of Scottish romantic landscape in the period, altered the younger 
avaient une prédilection pour les vues des Highlands et, ainsi que l'a précisé l'historien de l'art John Morrison, ils estimaient que les paysagistes qui ne s'attachaient pas à la représentation de ces montagnes reniaient non seulement les traditions artistiques nationales, mais remettaient aussi en question l'identité culturelle de l'Écosse ${ }^{10}$. Les académiciens n'approuvaient pas non plus les jeunes peintres qui prenaient pour modèles les artistes de l'Europe continentale ou qui allaient étudier à l'étranger plutôt que d'être formés à la Royal Scottish Academy ou dans l'atelier d'un peintre écossais (Morrison, 2003, p. 156).

Les relations des Glasgow Boys avec leurs collègues de la Royal Scottish Academy furent d'autant plus tendues que les jeunes artistes critiquaient ouvertement les académiciens qu'ils surnommaient les «gluepots» (Billcliffe, 2002, p. 30) en raison de leur utilisation excessive d'un vernis à base de bitume qui servait à donner à leurs tableaux un aspect de patine naturelle. Ils leur reprochaient aussi de ne pas peindre en plein air et de ne pas représenter fidèlement les paysages et les habitants de l'Écosse ${ }^{11}$. Ils n'appréciaient pas non plus la sentimentalité mièvre qui caractérisait la peinture de genre.

Avec leurs tableaux ayant pour thème des ouvriers agricoles au travail, les Glasgow Boys avaient pour ambition de représenter fidèlement l'Écosse contemporaine. L'Écosse était alors le deuxième pays le plus industrialisé d'Europe et de plus en plus d'Écossais allaient vivre dans les zones urbaines. Néanmoins, les zones rurales des Lowlands n'en étaient pas pour autant désertifiées à la fin du XIX ${ }^{\mathrm{e}}$ siècle, ainsi que l'ont rappelé Devine et Campbell :

[Even if] the experience of rural society in the later nineteenth century concerned a diminishing proportion of the people of Scotland [...] it was still the way of life found in a large part of the country. ("The Rural Experience», dans Fraser \& Morris, 1990, p. 46)

À l'instar des peintres naturalistes français qu'ils avaient côtoyés, ils s'étaient donné pour objectif de peindre en plein air l'homme contemporain sans apprêt ni idéalisation alors qu'il était occupé à des activités ordinaires.

artist's work as it hung in the exhibition. His assumption that Reid was attempting to join the ranks of romanticists and therefore that he would benefit from any such alteration was unchallenged by the RSA establishment.» (2003, p. 149)

10. Morrison affirme que : «The nineteenth-century creation of a Highland identity for the whole country was internalised by Scots. [...] Resistance to Highlandism was certainly viewed in artistic circles as a conscious attack on the entire underpinning of national cultural identity. » (2003, p. 147)

11. Selon Billcliffe, les Glasgow Boys estimaient que les paysages des peintres écossais «were all theatrical backcloths, survivals from an earlier age, painted without capturing the true value of the landscape, which could as much have been Switzerland as Scotland» (2002, p. 27). 
Les Glasgow Boys ne furent pas les premiers peintres écossais à prendre pour thème le travail puisque déjà, à la fin du XVIII ${ }^{\mathrm{e}}$ siècle, David Allan (1744-1796) avait réalisé une série de dessins consacrés aux différents corps de métiers présents dans les villes écossaises ${ }^{12}$. Au cours des années 1860 et 1870, George Reid et George Paul Chalmers (1833-1878) avaient peint plusieurs tableaux représentant des ouvriers agricoles écossais ${ }^{13}$. De prime abord, le choix du thème du travail agricole peut paraître surprenant pour des peintres de Glasgow, alors la deuxième plus grande agglomération de Grande-Bretagne et l'un des principaux centres industriels de l'Europe occidentale. Le travail industriel n'a cependant pas inspiré les Glasgow Boys, à l'exception de Lavery qui a célébré la prospérité de l'industrie navale de la ville dans Shipbuilding on the Clyde, peinture murale ornant la Glasgow City Chambers. Pour mieux comprendre cet intérêt pour la représentation du travail agricole, il convient de prendre en compte le contexte international.

À la fin du XIX ${ }^{\mathrm{e}}$ siècle, un grand nombre de peintres anglais et de l'Europe continentale ont peint des scènes paysannes. Les premiers tableaux des Glasgow Boys s'inscrivent dans le mouvement naturaliste, qui apparut tout d'abord en France avant de dominer brièvement l'art européen durant les années 1880 et 1890. Ce fut leur contact avec les naturalistes français lors de leur formation dans les ateliers parisiens et leur séjour dans la colonie d'artistes à Grez-sur-Loing qui incitèrent les Glasgow Boys à puiser leurs motifs dans les communautés villageoises. En France, à partir des années 1850, les peintres tels Millet, Breton et Léon Augustin Lhermitte (1844-1925) avaient suivi l'exemple de Gustave Courbet (18191877) et s'étaient attachés à peindre la vie quotidienne des paysans. Cet intérêt fut perpétué par Jules Bastien-Lepage et les jeunes naturalistes, qui apparurent sur la scène artistique vers la fin des années 1870. De retour en Écosse, les Glasgow Boys pouvaient suivre le développement de l'art français puisqu'ils avaient la possibilité de voir les œuvres des naturalistes dans les galeries des marchands d'art de Glasgow, notamment celles d'Alexander Reid (1854-1928) et de Craibe Angus (1830-1899), ainsi qu'à l'occasion des expositions du Glasgow Institute. À défaut d'être appréciés par les membres de la Royal Scottish Academy, les collectionneurs de Glasgow admiraient les œuvres des naturalistes français et avaient fait l'acquisition de plusieurs de leurs tableaux qu'ils prêtaient

12. Ces dessins sont désormais conservés à la National Gallery of Scotland. Voir notamment David Allan, An Edinburgh Fireman, D. 400, National Gallery of Scotland, Édimbourg; David Allan, Two Edinburgh Chimney Sweeps, D. 399, National Gallery of Scotland, Édimbourg.

13. En 1873, George Paul Chalmers peignit The End of the Harvest (collection particulière). Trois ans auparavant, Reid avait réalisé The Peat Gatherers (localisation inconnue). 
au Glasgow Institute. L'intérêt des amateurs pour les scènes paysannes explique aussi que les Glasgow Boys aient choisi de peindre les ouvriers agricoles des Lowlands.

Les tableaux des Glasgow Boys représentant les ouvriers agricoles des Lowlands se démarquèrent des productions artistiques écossaises contemporaines en raison de leur thème, mais aussi de leur style. Les toiles des peintres de scène de genre se caractérisaient par leur réalisme mimétique : ainsi, dans le tableau de Thomas Faed (1826-1900) intitulé From Hand to Mouth - He Was One of the Few Who Would Not Beg, tout dans la composition est peint avec minutie ce qui contraste avec les tableaux paysans des Glasgow Boys, tel Noon de George Henry qui juxtapose des parties rapidement brossées et des détails finement rendus. La peinture naturaliste des Glasgow Boys reposant sur des phénomènes de la vision était inspirée de celle de Jules Bastien-Lepage. Cet artiste jouissait d'une renommée considérable en Europe occidentale et surtout en GrandeBretagne où il séjourna à plusieurs reprises ${ }^{14}$. Seul Lavery put rencontrer Bastien-Lepage avant le décès de ce dernier en 1884, mais l'ensemble des Glasgow Boys lui vouait une grande admiration. Il suffit de comparer leurs tableaux paysans avec les toiles du maître français pour comprendre à quel point ils furent influencés par Bastien-Lepage. Face aux peintures académique et romantique qui prévalaient en Écosse, les premiers tableaux des Glasgow Boys ont ainsi ouvert une nouvelle voie, celle d'une peinture naturaliste alliant la précision du dessin académique à la liberté de la touche et à l'apport du plein air sur la lumière et la couleur empruntés à l'impressionnisme ${ }^{15}$. Cet intérêt pour la couleur et l'influence de l'art français marqua profondément les générations suivantes de peintres écossais et plus précisément le groupe des Colourists.

En dépit de leurs affinités stylistiques et thématiques avec les œuvres des naturalistes français, les scènes de la vie paysanne écossaise peintes par les Glasgow Boys représentent une réalité propre à la majeure partie des zones rurales des Lowlands et évoquent quelques-unes des principales caractéristiques du travail agricole dans la région à la fin du XIX ${ }^{\mathrm{e}}$ siècle. Durant les années 1880, les peintres de l'école de Glasgow se sont souvent retrouvés pour peindre dans les villages de Cockburnspath, dans le comté de Berwick, et de Kirkcudbright, dans le comté de Dumfries et Galloway,

14. Bastien-Lepage alla à quatre reprises en Grande-Bretagne. Il se rendit à Londres pour la première fois en 1879 afin de réaliser le portrait du Prince de Galles. Il retourna dans cette ville en 1880, en 1881 et en 1882 .

15. Au même moment, William McTaggart (1835-1910) peignait lui aussi des tableaux allant à l'encontre de la peinture académique. Ces toiles représentant des paysages de la côte ouest de l'Écosse ont souvent été comparées aux œuvres des peintres impressionnistes. 
où ils furent à l'origine de deux colonies d'artistes. Afin de peindre les tableaux les plus réalistes et les plus représentatifs possibles des ouvriers agricoles des Lowlands, Guthrie alla même jusqu'à s'installer pendant plusieurs années à Cockburnspath où il reçut régulièrement la visite de Walton, Crawhall, Melville, Henry et Whitelaw Hamilton (1860-1930). Ce fut dans le village de Kirkcudbright où Hornel avait son atelier, que celui-ci allait peindre en plein air en compagnie de Henry et de Guthrie.

Si l'on considère l'ensemble des tableaux que les Glasgow Boys ont consacré au travail agricole, on peut constater que deux thèmes sont récurrents : ils ont réalisé un grand nombre de toiles dédiées aux travaux des champs et ont aussi souvent peint des paysans travaillant dans leur potager. Chacune de ces œuvres peut être associée aux Lowlands et rappelle la spécificité de l'agriculture écossaise à cette époque. Certains titres de tableaux tels Berwickshire Fieldworkers et In the Town Crofts, Kirkcudbright, de même que quelques éléments de la composition, tels le couvre-chef ${ }^{16}$ que portent les ouvrières agricoles dans le premier tableau et la silhouette distinctive de l'église de Kirkcudbright dans la seconde toile, permettent d'associer les scènes représentées à un lieu précis. Le tableau de Guthrie intitulé $A$ Hind's Daughter ne fait pas référence à un comté ou à un village, mais le terme «hind» évoque une des caractéristiques de l'agriculture des Lowlands et rappelle que dans ces comtés, le travail agricole reposait sur des traditions perpétuées de génération en génération depuis des siècles. Dans les comtés du sud de l'Écosse, l'agriculture avait beaucoup évolué depuis la fin du XVIII ${ }^{\mathrm{e}}$ siècle, elle s'était modernisée mais préservait encore de nombreuses traditions. Au moment où les Glasgow Boys réalisèrent leur série de tableaux paysans, dans certains comtés, les ouvriers agricoles continuaient à travailler en famille comme leurs ancêtres l'avaient fait et, suivant la coutume, les filles d'une certaine catégorie d'ouvriers étaient encore destinées à travailler dans les champs. Dans A Hind's Daughter Guthrie a choisi de représenter la fille d'un ouvrier agricole appartenant à la catégorie des «hinds». Dans les Lowlands, on distinguait alors trois groupes d'ouvriers agricoles : il y avait les ouvriers recrutés à la journée, les ouvriers célibataires qui avaient un contrat de plusieurs mois et les ouvriers mariés, appelés les «hinds», vivant avec leur famille dans une ferme qu'ils occupaient gratuitement si en échange ils trouvaient une femme ou une jeune fille prête à participer aux travaux des champs durant les périodes de récoltes ${ }^{17}$. Le plus souvent, c'était une des filles de l'ou-

16. Le couvre-chef que portent les ouvrières agricoles dans le tableau de Berwickshire Fieldworkers était connu sous le nom de ugly et était traditionnellement porté par les femmes du comté de Berwick.

17. Alastair Orr a précisé : «Farm servants as a group consisted broadly of three subdivisions: married ploughmen (called 'hinds' in the Lothians') living in a cottage attached to the farm; unmarried ploughmen boarded in the 
vrier agricole, comme celle figurant dans le tableau de Guthrie, qui travaillait à cette occasion. Ce système était en vigueur depuis des siècles en Écosse mais, au moment où Guthrie peignit $A$ Hind's Daughter cette pratique avait déjà fait l'objet de critiques et commençait à disparaître. Néanmoins, jusqu'à la Première Guerre mondiale, cette tradition resta ancrée dans les familles travaillant dans les comtés de Berwick et de l'East Lothian, ainsi que l'a rappelé Alastair Orr (dans Devine, 1984, p. 33).

Lorsque l'on observe les tableaux paysans des Glasgow Boys on remarque que les personnages travaillant dans les champs ou dans les potagers sont majoritairement des femmes ou des jeunes filles. Dans Berwickshire Fieldworkers de Walton, les cinq personnages représentés aux champs sont des femmes, tandis que dans Hop-Pickers Returning, parmi les quatre personnages peints par Mann, figurent deux jeunes femmes et une fillette. Ceci reflète une autre tradition écossaise et une des principales caractéristiques du travail agricole dans les Lowlands. Durant les années 1870, les femmes constituaient près de $26 \%$ de la main d'œuvre agricole de cette région et, ainsi que l'a précisé Orr, dans certains comtés, elles représentaient parfois jusqu'à 46\% des ouvriers agricoles (dans Devine, 1984, p. 34). Entre 1851 et 1911, le nombre de femmes travaillant dans l'agriculture augmenta de plus de $30 \%$, alors qu'en Angleterre leur nombre ne cessa de décliner. Plusieurs facteurs permettent d'expliquer ce phénomène. Tout d'abord, les femmes avaient toujours occupé une place importante dans l'agriculture de la région, certaines tâches telles la traite et la fabrication de fromages leur étaient traditionnellement réservées dans les comtés du sud-ouest. Dans ceux du sud-est, la spécialisation dans la culture du navet et de la pomme de terre ne fit qu'accroître la main d'œuvre féminine puisque pour ce type de récolte les fermiers estimaient que les femmes étaient plus adaptées (Orr, dans Devine, 1984, p. 43). Ensuite, le recrutement des femmes présentait un intérêt financier, leur salaire étant bien moins élevé que celui des hommes. Enfin, beaucoup d'hommes délaissèrent le travail de la terre dès lors que les industries lourdes commencèrent à se développer et pour pallier le départ des hommes, un plus grand nombre de femmes fut recruté. Même si un certain nombre d'entre elles quittèrent les zones rurales pour aller vivre en ville et travailler dans l'industrie, il n'y eut pas de pénurie de main d'œuvre féminine jusqu'à la fin du XIX ${ }^{\mathrm{e}}$ siècle puisque des femmes originaires des Highlands ou d'Irlande venaient spécialement dans les zones rurales des Lowlands pour trouver du travail. Dans les fermes, elles

farmer's house, and domestic servants who were usually women.» («Farm Servants and Farm Labour in the Forth Valley and South-East Lowlands», dans Devine, 1984, p. 30) 
étaient embauchées pour effectuer tous types de travaux à l'exception des tâches nécessitant l'utilisation des chevaux, celles-ci étant réservées aux hommes ${ }^{18}$. Cette réalité propre aux Lowlands est apparente dans les œuvres des Glasgow Boys. Entre autres, dans leurs tableaux intitulés Noon, A Cottar's Garden, Hop-Pickers Returning et Potato Planting on voit des femmes ou des jeunes filles alors qu'elles sont occupées à faire paître le bétail, à récolter des légumes, à cueillir du houblon et à planter des pommes de terre; elles sont aussi représentées en train de sarcler, de biner et de moissonner.

Ce qui ressort de leurs œuvres c'est avant tout que l'ensemble des habitants des communautés rurales participait aux travaux agricoles : les peintres de Glasgow ont représenté beaucoup d'ouvrières, mais pas seulement puisqu'ils ont également peint des hommes et des enfants dans les champs. Dès lors qu'ils étaient en âge de travailler, les enfants se voyaient attribuer des tâches telles que surveiller un troupeau de vaches comme on peut le constater dans le tableau de Henry intitulé Noon. Le vieil homme représenté par Henry dans The Hedgecutter rappelle aussi que même les personnes d'un âge avancé continuaient à travailler dans les champs. Si l'on considère l'ensemble des tableaux que les Glasgow Boys ont consacré à leurs compatriotes vivant dans les communautés agricoles des Lowlands, on remarque que ces derniers sont le plus souvent représentés en plein labeur, suggérant ainsi que leur vie était centrée sur le travail et qu'il y avait peu de place pour d'autres activités. Ils peignirent quelques tableaux traitant d'autres sujets, mais ceux-ci furent peu nombreux et concernèrent surtout les enfants. Les habitants des zones rurales sont donc présentés comme étant toute leur vie des travailleurs acharnés, restés attachés aux traditions. Les peintres de Glasgow ont aussi montré que ce travail assidu profitait à l'ensemble des habitants de la communauté. Les personnages représentés dans leurs scènes champêtres portent des vêtements en bon état, ils ne semblent pas souffrir de la faim et, même si leurs tâches étaient dures et intenses, ils ne paraissent pas non plus épuisés. Ceci démarque les tableaux des Glasgow Boys de ceux de leurs collègues français qui, dans leurs toiles, ont souvent dénoncé les conditions de travail ${ }^{19}$. À la fin du XIX ${ }^{\mathrm{e}}$ siècle, dans les Lowlands, les ouvriers agricoles travaillaient dans des conditions difficiles, les femmes pouvaient avoir des journées de seize heures, pour des salaires souvent modiques.

18. Selon Devine : "[Women's duties] were to perform all the 'normal operations' of the fields and those concerned with the use of 'smaller implements' not worked by horses.» (1984, "Women Workers, 1850-1914», p. 98-99).

19. Déjà les peintres réalistes français avaient critiqué à travers leurs toiles le système économique contemporain. Suivant l'exemple de Gustave Courbet (1819-1877), qui ne cachait pas son engagement politique, les réalistes ont notamment dénoncé les conditions de travail et de vie des ouvriers. 
Pourtant, les peintres suggèrent que, grâce à leur labeur, les paysans étaient en mesure de subvenir aux besoins de leur famille. Dans A Hind's Daughter, Guthrie a peint une fillette dans le potager que le propriétaire foncier avait attribué à son père pour son utilisation personnelle et, en arrière-plan, il est possible de distinguer la maison que la famille de la fillette pouvait occuper en échange de leur travail dans les champs. Les enfants des ouvriers agricoles figurant dans les œuvres des Glasgow Boys n'ont rien en commun avec ceux vêtus de haillons représentés dans les tableaux intitulés Pas mèche et Pauvre fauvette, peints par Bastien-Lepage lorsqu'il séjournait dans son village natal de Damvillers, dans la Meuse. Les toiles des Glasgow Boys ne manifestent pas d'engagement politique de la part des artistes; ces derniers célèbrent dans leurs tableaux le travail intensif de leurs compatriotes qui contribuait à la renommée et à la prospérité de l'agriculture écossaise ${ }^{20}$.

Ces tableaux proposent donc une vision des Écossais très différente de celle véhiculée par les œuvres d'un peintre tel que Kenneth Macleay (1802-1878). Cet aquarelliste victorien fut très populaire pour ses portraits représentant des habitants des Highlands vêtus d'un kilt. Dans ses œuvres, les modèles incarnent force, fierté et esprit guerrier, des qualités qui étaient alors attribuées à l'ensemble des Écossais, ainsi que l'a rappelé Pittock dans son ouvrage intitulé Scottish Nationality ${ }^{21}$. Plusieurs Glasgow Boys peignirent également des paysages et, contrairement à leurs contemporains écossais, ils préférèrent représenter des vues des Lowlands. Ces paysages et les scènes rurales des peintres de Glasgow peuvent être perçus comme une alternative à la manière dont les peintres représentaient l'Écosse depuis plus d'un demi-siècle.

Avec leur série de tableaux consacrés au monde rural des Lowlands, les peintres de l'école de Glasgow connurent un immense succès auprès des collectionneurs et des critiques en Grande-Bretagne et sur le continent européen. Même les membres de la Royal Scottish Academy reconnurent leur talent et, en 1888, Guthrie fut le premier Glasgow Boy à être élu membre associé de cette institution. Par la suite, les principaux représentants de l'école de Glasgow devinrent académiciens et, en 1902, Guthrie en fut même élu président.

20. Devine a noté que l'agriculture écossaise a elle aussi ressenti la crise agraire qui frappa la Grande-Bretagne à partir des années 1870, mais il a précisé que : «Scottish farm workers were not seriously affected by the decline in agricultural prices between 1870 and 1900. On the contrary, over the period as a whole, real incomes rose for many workers. » (1984, p. 246).

21. Pittock a écrit : "The idea of the primitive, picturesque Scot as a giant was one [...] found in many Victorian postcards and popular depictions of vast and hairy Scots soldiers. [...] Emphasis was repeatedly put on the 'strong', 'hardy', 'sturdy' and 'robust' qualities of Scots. » (2001, p. 86) 
Les tableaux représentant les ouvriers agricoles furent à l'origine d'une révolution esthétique dans la peinture écossaise et ont marqué d'une forte empreinte la création des peintres de la première moitié du xx siècle. Ces œuvres constituent également un précieux témoignage des pratiques agricoles dans les Lowlands à la fin du XIX ${ }^{\mathrm{e}}$ siècle. Avec leurs scènes paysannes, les Glasgow Boys ont proposé une représentation de l'Écosse et des Écossais très différente de l'image véhiculée par les tableaux des peintres depuis les années 1820. Toutefois leur vision ne fut pas plus représentative de l'ensemble du pays puisque leurs œuvres montrent uniquement la vie de leurs contemporains dans les zones rurales des comtés du sud et n'évoquent pas les conditions difficiles. Les Glasgow Boys ont célébré le labeur acharné de leurs compatriotes tout au long de leur existence et ont rendu plus particulièrement hommage à la participation des femmes. Ces tableaux suggèrent que la qualité principale des Écossais était leur tempérament travailleur; le travail est présenté comme une valeur traditionnelle et fondamentale de la société rurale écossaise.

\section{Références bibliographiques}

\section{Ouvrages}

Billaliffe Roger, 2002, The Glasgow Boys. The Glasgow School of Painting 1875-1895, Londres, John Murray.

- (éd.), 2010, Pioneering Painters: The Glasgow Boys 1880-1900, Glasgow, Glasgow Museums.

Caw James, 1908, Scottish Painting Past and Present, 1620-1908, Édimbourg, Jack.

Devine Thomas Martin, 1999, The Scottish Nation, 1700-2000, Londres, Penguin Books.

- (éd.), 1984, Farm Servants and Labour in Lowland Scotland, 1770-1914, Édimbourg, John Donald Publishers.

Fraser William Hamish \& Morris R.J. (éds), 1990, People and Society in Scotland. Volume 2 : 1830-1914, Édimbourg, John Donald Publishers.

Hardie William, 1990, Scottish Painting, 1837-1939, Londres, Studio Vista.

Lemoine Serge \& Lobstein Dominique, 2007, fules Bastien-Lepage (18481884), Paris, Musée d'Orsay.

Macmillan Duncan, 2000, Scottish Art, 1460-2000, Édimbourg, Mainstream.

Martin David, 1897, The Glasgow School of Painting, Londres, Bell.

MaConkey Kenneth, 2010, John Lavery a Painter and His World, Édimbourg, Atelier Books. 
Morrison John, 2003, Painting the Nation. Identity and Nationalism in Scottish Painting, 1800-1920, Édimbourg, Edinburgh University Press.

PitTock Murray, 2001, Scottish Nationality, Basingstoke, Palgrave.

Smout Thomas Christopher, 1972, A History of the Scottish People 15601830, Grande-Bretagne, Fontana.

Walsh Jean \& Stevenson Hugh, 2010, Introducing the Glasgow Boys, Glasgow, Glasgow Museums.

\section{Tableaux cités}

Quelques-uns des tableaux cités dans cet article peuvent être consultés sur les sites internet indiqués.

Bastien-Lepage Jules, Pauvre Fauvette, 1881, Glasgow Art Gallery and Museum, Glasgow. Disponible sur <www.the-athenaeum.org/art/ detail.php?ID=50297>.

_- Pas mèche, 1882, National Gallery of Scotland, Édimbourg. Disponible sur <www.nationalgalleries.org/collection/artists-a-z/b/artist/ jules-bastien-lepage/object/pas-meche-nothing-doing-ng-1 133>.

FAED Thomas, From Hand to Mouth - He Was One of the Few Who Would Not $\mathrm{Beg}$, 1879, collection particulière. Disponible sur <www.victorianweb. org/painting/faed/paintings/1.html>.

Guthrie James, A Hind's Daughter, 1883, National Gallery of Scotland, Édimbourg. Disponible sur <www.bbc.co.uk/arts/yourpaintings/ paintings/a-hinds-daughter-210151>.

Henry George, A Cottar's Garden, 1885, Broughton House, The National Trust for Scotland. Disponible sur <www.the-athenaeum.org/art/ detail.php?ID $=50371>$.

- The Hedgecutter, 1885, collection particulière. Disponible sur <www. bbc.co.uk/arts/yourpaintings/paintings/the-hedge-cutter>.

—, Noon, 1885, collection particulière. Disponible sur < http://www.theathenaeum.org/art/detail.php?ID=50373>.

Hornel Edward Atkinson, In the Town Crofts, Kirkcudbright, 1885, collection particulière. Disponible sur <www.the-athenaeum.org/art/detail.php ?ID=50391>.

—, Potato Planting, 1886, coll. particulière. Disponible sur <www.artistsfootsteps.co.uk/artists_a_z.asp?ID $=66+\&$ loadType $=2 \&$ offset $=5>$.

Lavery Sir John, Shipbuilding on the Clyde, 1889-1901, Glasgow City Chambers, Glasgow. Disponible sur <www.bbc.co.uk/arts/yourpaintings/ paintings/shipbuilding-on-the-clyde-84782>.

Mann Alexander, Hop-Pickers Returning, 1883, Hunterian Art Gallery, University of Glasgow, Glasgow. Disponible sur <www.bbc.co.uk/ arts/yourpaintings/paintings/hop-pickers-returning $>$. 
ÉTUDES ÉCOSSAISES 18

Walton Edward Arthur, Berwickshire Fieldworkers, 1884, Tate Britain, Londres. Disponible sur <www.tate.org.uk/art/artworks/walton-ber wickshire-field-workers-t03447>. 